\title{
La degradación de los bosques de altura del centro de Argentina reduce su capacidad de almacenamiento de agua
}

\author{
María Poca ${ }^{1,2,10}$; Ana M. Cingolani ${ }^{12}$; Diego E. Gurvich ${ }^{12,2}$; Juan I. Whitworth-Hulse ${ }^{1}$ \\ \& Valentina Saur Palmieri ${ }^{2}$ \\ ${ }^{1}$ Instituto Multidisciplinario de Biología Vegetal (CONICET-Universidad Nacional de Córdoba), Córdoba, Argentina. \\ ${ }^{2}$ Facultad de Ciencias Exactas, Físicas y Naturales, Universidad Nacional de Córdoba, Córdoba, Argentina.
}

\begin{abstract}
Resumen. La capacidad del suelo de almacenar y regular el flujo de agua depende en gran medida de su su tasa de infiltración y profundidad. Los disturbios como el pastoreo y el fuego son moduladores fuertes del sistema vegetación-suelo, ya que son capaces de alterar la tasa de infiltración y la profundidad del suelo. En sistemas montanos estacionales, esto repercute sobre la capacidad de liberar lentamente el agua almacenada hacia el caudal de los arroyos. Nos propusimos indagar si las variaciones en la estructura de la vegetación, debidas en su mayoría al pastoreo y al fuego, de los bosques de altura de Polylepis australis Bitter de las sierras de Córdoba se asocian a variaciones en las propiedades del suelo en general y, en particular, a aquellas que regulan la capacidad de almacenar agua. Para abarcar la variabilidad completa de estructura de la vegetación seleccionamos 28 sitios distribuidos en tres establecimientos con distintos manejos, y restringimos las variaciones topográficas. No detectamos asociaciones entre la estructura de la vegetación y la topografía; es decir, en general, logramos restringir el efecto topográfico. Por otro lado, aquellos sitios con vegetación más estructurada presentaron suelos con menor densidad aparente y mayor contenido de materia orgánica y capacidad de campo. En relación a la capacidad de almacenamiento de agua, los suelos más profundos y con mayor tasa de infiltración fueron los menos densos, con mayor contenido de materia orgánica y mayor capacidad de campo, asociados a una vegetación más estructurada. Es decir, mientras más conservado se encuentra el sistema vegetación-suelo de los bosques de $P$. australis, mayor cantidad de agua puede ingresar al suelo y ser almacenada. En consecuencia, las alteraciones del sistema vegetación-suelo, dadas principalmente por el pastoreo y el fuego, reducen la capacidad de almacenar agua de los bosques de altura del centro de la Argentina.
\end{abstract}

[Palabras clave: Polylepis australis, tasa de infiltración, profundidad del suelo, estructura de la vegetación, suelo, montañas, pastoreo, fuego, disturbio, ecohidrología]

\begin{abstract}
Aвstract. The degradation of highland woodlands of central Argentina reduces their soil water storage capacity. Soil water storage capacity and flow regulation relies mostly upon infiltration rate and soil depth. Disturbs such as grazing and fire strongly modulate the vegetation-soil system, and are capable of altering the infiltration rate and soil depth, as well. In mountain seasonal ecosystems, this impacts on soil capacity to slowly release water into streams. We aimed at analyzing if changes in vegetation structure and soil properties, mainly as a consequence of grazing and fire, of highland Polylepis australis Bitter woodlands of Córdoba mountains, also include alterations in general soil properties and, in particular, in those related to soil water storage capacity. In order to encompass the complete variability in vegetation structure we selected 28 sites in paddocks with different managements, restricting topographic variations. We did not find associations between vegetation structure and topographic variables, which indicates we could restrict the topographic variability. Sites with more structured vegetation had soils with lower soil bulk density and higher soil organic matter content and field capacity. Regarding soil water storage capacity, deeper soils with higher infiltration rates were those with more dense soils and higher organic matter content and field capacity, associated to more structured vegetation. This means that while vegetation-soil system of $P$. australis woodlands is more conserved, the soil presents more opportunities of infiltrating and storing water. Consequently, alterations on the vegetation-soil system, mainly given by grazing and fire, reduce soil water storage capacity of highland woodlands of central Argentina.
\end{abstract}

[Keywords: Polylepis australis, infiltration rate, soil depth, vegetation structure, soil, mountains, grazing, fire, disturbs, ecohydrology]

Editor asociado: Gervasio Piñeiro

\ pocamaria@gmail.com
Recibido: 21 de diciembre de 2016

Aceptado: 8 de junio de 2017 


\section{INTRODUCCIÓN}

La presencia de vegetación estructurada, entendida como aquella con mayor área foliar, biomasa y cobertura, así como mayor estratificación vertical y superposición de estratos, tiene efectos positivos sobre las propiedades edáficas. La vegetación estructurada permite la acumulación de broza y un mayor ingreso de materia orgánica al suelo. También aumenta la aireación del suelo a través del desarrollo de la rizósfera y de la proliferación de la biota del suelo. En conjunto, todo ello promueve las oportunidades de infiltración del agua en las primeras capas del suelo y la percolación a las capas más profundas (Neary et al. 2009; Bonell et al. 2010; Germer et al. 2010). Además, la vegetación estructurada previene la erosión eólica e hídrica, evitando la pérdida de horizontes y reducción en la profundidad del suelo (Zhang et al. 2004).

Los disturbios como el pastoreo y el fuego son moduladores fuertes del paisaje, y poseen el potencial para reducir la estructura de la vegetación y alterar las propiedades físicoquímicas del suelo (Milchunas and Lauenroth 1993; Belsky and Blumenthal 1997; Bernardi et al. 2016). Los cambios en la estructura de la vegetación causados por estos disturbios pueden ser tan drásticos como para convertir bosques en pastizales $u$ otras coberturas de menor complejidad estructural. Al disminuir la cobertura vegetal también se reduce el aporte de broza, lo cual disminuye el ingreso de materia orgánica al suelo (Abdel-Magid et al. 1987; Yong-Zhong et al. 2005; Altesor et al. 2006). Debido al pisoteo del ganado, la densidad aparente (i.e., peso seco de suelo por unidad de volumen) y la compactación del suelo tienden a aumentar y la capacidad de retención de agua a disminuir (Boone Kauffman et al. 2004; Yong-Zhong et al. 2005; Altesor et al. 2006). En conjunto, estas alteraciones en la estructura de la vegetación y en las propiedades edáficas pueden ir acompañadas de una disminución en la tasa de infiltración del agua en el suelo (AbdelMagid et al. 1987; Boone Kauffman et al. 2004; Yong-Zhong et al. 2005). Además, en sistemas montanos, el pastoreo y el fuego a menudo producen pérdidas de las partículas finas del suelo por erosión, y ante condiciones severas de disturbio son promotores de una pérdida significativa del perfil (Pimentel et al. 1995). Cuando se reduce la profundidad de un suelo también decrece la cantidad máxima de agua que puede almacenar. En este sentido, la tasa de infiltración y la profundidad cumplen un rol determinante en la capacidad del suelo de almacenar y regular el flujo de agua (NoyMeyr 1973). Por lo tanto, el disturbio, al alterar la estructura de la vegetación y las propiedades físico-químicas del suelo, incluidas la tasa de infiltración y profundidad del suelo, altera también su capacidad para almacenar agua. En última instancia, en sistemas estacionales, esto repercutirá en la capacidad del suelo de retener el agua hasta la estación seca y de alimentar a los arroyos en dicha época (Bruijnzeel 1988, 2004; Roa-García et al. 2011) a través de flujo por desplazamiento y flujo preferencial (Beven and Germann 2013).

Numerosos trabajos realizados en el piso superior de las sierras de Córdoba (centro de la Argentina), han indicado que el pastoreo por ganado doméstico junto al fuego asociado al manejo son modeladores clave de la estructura de la vegetación (Renison et al. 2006, 2010, 2015; Cingolani et al. 2008, 2014). Dicho manejo del sistema es capaz de disminuir el crecimiento, e incluso aumentar, la mortalidad de los individuos de Polylepis australis Bitter, el árbol dominante de la región; esto reduce la cobertura de dichos bosques (Teich et al. 2005; Cingolani et al. 2008, 2014; Giorgis et al. 2010; Renison et al. 2010). Además, el ganado, en combinación con el fuego, promueve la apertura de los pajonales en mata, lo que da lugar a céspedes de pastoreo. Ante condiciones de sobrepastoreo se generan pavimentos de erosión o pedregales donde la cobertura vegetal se reduce al mínimo y prevalece la roca expuesta debido a la pérdida parcial o total del suelo (Cingolani et al. 2003, 2008, 2013, 2014). Si bien la profundidad del suelo es naturalmente variable debido a la microrugosidad de la roca madre y a la topografía, se ha encontrado que el disturbio actúa de manera sinérgica con estas variaciones naturales, alterando las propiedades edáficas (Cingolani et al. 2008, 2013). De hecho, se ha indicado que el disturbio, además de reducir la profundidad, es capaz de promover la pérdida de la calidad del suelo al compactarlo, disminuir el contenido de materia orgánica y aumentar levemente su salinidad y acidez (Renison et al. 2010). Asimismo, en un trabajo reciente para el área de estudio, Cingolani et al. (2015) sugirieron que la profundidad del suelo podría jugar un papel clave en la liberación de agua al caudal de los arroyos en la estación seca.

En este contexto, nos propusimos investigar si las variaciones en la estructura de la 
vegetación debidas al pastoreo y al fuego de los bosques de altura de las sierras de Córdoba se asocian a variaciones en las propiedades del suelo en general y, en particular, a aquellas que regulan la capacidad de almacenar agua. Para ello, buscamos seleccionar sitios que incluyeran toda la variación fisonómica existente, abarcando establecimientos con distintas historias de uso y restringiendo las variaciones topográficas. Como objetivos específicos nos planteamos: a) describir los sitios en término de las variaciones en las características de la vegetación, del suelo y la topografía (ésta última para corroborar que realmente no varía) y seleccionar, a través de métodos multivariados, las variables más representativas para cumplir con los objetivos subsiguientes; b) analizar las relaciones entre las características de la vegetación, las propiedades edáficas superficiales, y la topografía, y c) analizar si las características de la vegetación, las propiedades edáficas superficiales y la topografía se asocian a variaciones en la tasa de infiltración y la profundidad del suelo, como indicadores de la capacidad de almacenamiento de agua en el suelo de estos ecosistemas. Esperamos que las variaciones en las características de la vegetación se asocien con variaciones en el suelo, incluyendo las variables indicadoras de la capacidad de almacenamiento. Asimismo, considerando el criterio utilizado para seleccionar los sitios, esperamos que el suelo y la vegetación no se asocien con las variables topográficas. Sobre la base de una gran cantidad de evidencias contenidas en estudios previos (citados más arriba) y en el manejo de los establecimientos en los que se ubicaron los sitios (ver detalles en la sección Área de estudio), asumimos que si efectivamente no encontramos relación entre la topografía y las variables de vegetación y suelo, podemos atribuir la variación (o gran parte de ella) en la vegetación y los suelos a los efectos del disturbio a largo plazo.

\section{Materiales y Métodos}

\section{Área de estudio}

Realizamos este trabajo en los bosques de altura de las Sierras Grandes de Córdoba, en el centro de la Argentina. Las Sierras Grandes tienen una orientación norte-sur; su pico más alto es el cerro Champaquí (2789 m s.n.m.). Sin embargo, la mayor parte del área tiene una altitud que oscila entre 2000 y 2300 m s.n.m. Consiste en una altiplanicie disectada, la "Pampa de Achala", remanente de una peneplanice cristalina antigua (Figura Aa del Apéndice ). El paisaje es muy heterogéneo, tanto en su topografía como en su cobertura vegetal. Consiste en lomas y planicies con valles suaves y profundos, y suelos Molisoles con altos contenidos de materia orgánica, derivados del lavado de la roca granítica y depósitos eólicos de textura fina (Cabido et al. 1987). La temperatura media del invierno es $5^{\circ} \mathrm{C}$, mientras que la del verano es $11.4{ }^{\circ} \mathrm{C}$, y no hay meses libres de heladas (Colladon 2004). La precipitación media anual es 900 $\mathrm{mm}$, concentrados en los meses más cálidos (entre octubre y abril) (Colladon 2014). La evapotranspiración potencial fue estimada en $576 \mathrm{~mm}$ (Cingolani et al. 2015).

El paisaje se conforma por un mosaico de vegetación con estructura variable, que comprende desde bosques de $P$. australis y pajonales con distinto grado de apertura hasta pedregales y pavimentos de erosión con sólo pequeños parches de suelo remanente (Cingolani et al. 2003, 2008, 2013, 2014; Renison et al. 2006, 2010). El pastoreo por ganado doméstico y el fuego asociado al manejo impactaron fuertemente sobre el sistema y causaron cambios estructurales, en algunos casos poco reversibles (Cingolani et al. 2003, 2008, 2013, 2014). Esto trajo aparejado un deterioro del capital natural (Aronson et al. 2007; Renison et al. 2010). Por un lado, se estima que $20 \%$ del área perdió el perfil completo del suelo a lo largo de los últimos 400 años de historia de pastoreo doméstico; en el presente está cubierto por roca expuesta (Cingolani et al. 2008, 2013). Por el otro, se redujo considerablemente la cobertura de bosques de $P$. australis (Teich et al. 2005; Renison et al. 2006, 2015; Cingolani et al. 2008). De esta manera, hoy en día, la herbivoría y el fuego generan y mantienen diferentes tipos de parches de vegetación (Cingolani et al. 2008, 2013 , 2014). Las variaciones en la intensidad del disturbio actual y pasado de distintos sitios pueden deberse tanto a los distintos manejos de los potreros como al uso diferencial que hacen los animales de los sitios de un mismo potrero (von Müller et al. 2017). En paisajes de lomas y laderas, cuando las áreas han tenido un uso histórico leve, con poco fuego y baja presión de pastoreo, hoy se encuentra una cobertura boscosa de $P$. australis relativamente alta, tanto en posiciones topográficas altas como bajas. En áreas o potreros con una mayor intensidad de uso pasado encontramos menos bosques, a menudo restringidos a posiciones topográficas más bien bajas. En 
cambio, en su reemplazo encontramos una mayor cobertura de pajonales y céspedes, así como algunos parches de pedregales y pavimentos de erosión. Por último, en lugares que sufrieron una presión de uso muy intenso prácticamente no hay bosques, sólo árboles aislados; además, encontramos un mosaico de pedregales y pavimentos de erosión, con parches de céspedes y algunos pajonales muy degradados (Cingolani et al. 2003, 2004, 2008, 2013, 2014; Renison et al. 2006, 2010). En situaciones que tuvieron una presión alta de uso en el pasado, pero en las que recientemente se excluyó el disturbio, los bosques se comenzaron a recuperar (Teich et al. 2005; Giorgis et al. 2010); en estos casos, la velocidad de recuperación del estrato arbóreo depende de la proximidad a árboles semilleros (Torres et al. 2008; Cingolani et al. 2014). Por este motivo, en áreas bajo exclusión se pueden encontrar, además de bosques en recuperación, matorrales de otras especies leñosas, pajonales cerrados y helechales. También se encuentran pedregales y pavimentos, ya que en esos sitios la recuperación de la cobertura vegetal es muy lenta (aunque muchas veces en estos pedregales se observan individuos de $P$. australis que germinan y empiezan a crecer en pequeñas fisuras [Cingolani et al. 2014]). En el año 1997, parte de esta área fue declarada Parque Nacional Quebrada del Condorito. En algunas zonas del Parque se excluyó el ganado doméstico para favorecer la recuperación de la vegetación (APN 2007).

Para este trabajo seleccionamos 28 sitios en un área de $\sim 50$ ha, en un paisaje de lomas y laderas a 2000 m s.n.m., con un punto central en $31^{\circ} 37^{\prime}$ S - $64^{\circ} 45^{\prime} \mathrm{W}$ (Figura Aa y Ab del Apéndice). Los sitios, de 10x10 m, abarcaron la variabilidad completa en la estructura de la vegetación existente en el área de estudio, desde bosques hasta pedregales. Para ello fue necesario abarcar zonas dentro del Parque Nacional sin pastoreo desde hace alrededor de 20 años y fuera del mismo en dos establecimientos privados con uso ganadero en la actualidad. Ninguno de los tres establecimientos ha experimentado fuegos en los últimos 20 años dentro del área en la cual seleccionamos los sitios. Asimismo, evidencias indirectas sugieren que, con anterioridad a la creación del Parque Nacional, los tres establecimientos tuvieron distinto uso histórico. El uso más leve fue el del área incluida dentro del Parque Nacional, fue intermedio en el establecimiento 1 y alto en el establecimiento 2 (Cingolani et al. 2004, 2008) (Figura Ac del Apéndice). En todos los sitios, o muy próximos a ellos, se podían encontrar individuos de $P$. australis. Los 28 sitios se seleccionaron procurando que tuviesen pendientes moderadas, posiciones topográficas intermedias y orientaciones sur, sureste y suroeste.

\section{Topografía}

Medimos seis variables topográficas en los 28 sitios para corroborar que logramos restringir la topografía de modo que las variaciones en vegetación y suelos se puedan interpretar como efectos del disturbio. Medimos la altitud (m s.n.m.) utilizando un sistema de posicionamiento global (GPS) que refleja la posición topográfica de los sitios en el paisaje, ya que el rango de variación fue menor a 60 m. La pendiente $(\%)$ y la orientación $\left(^{\circ}\right)$ se midieron con un clinómetro y una brújula, respectivamente. Como un indicador de la rugosidad medimos la distancia $(\mathrm{cm})$ desde la roca o elevación de suelo más alta del sitio hacia la parte adyacente más baja. Estimamos el índice de insolación diario $\left(^{\circ}\right)$ como la diferencia entre el plano horizontal y el ángulo que impide la incidencia del sol en el sitio debido a montañas o rocas tanto hacia el este como el oeste (Cingolani et al. 2008, 2014). Además, calculamos un índice de insolación anual a partir del coseno de la orientación multiplicado por la pendiente. En este último caso los valores negativos indican orientaciones sur, mientras que los valores cercanos a cero indican orientaciones este $u$ oeste (o situaciones con muy baja pendiente) y los valores positivos indican orientación norte. Finalmente, medimos a campo el área de acumulación, o área de contribución de agua hacia un punto dado (Gómez-Plaza et al. 2001) de cada sitio. Para ello tomamos como punto de referencia al área total del sitio de 10x10 m. Es decir, estimamos a campo toda el área que contribuye agua a cualquier punto del sitio. Se recorrió con un GPS para registrar el perímetro del área de contribución de agua hacia dicho sitio. Luego, ingresamos el perímetro al Google Earth Pro ${ }^{\circledR}$ para calcular el área de acumulación de cada sitio $\left(\mathrm{m}^{2}\right)$.

\section{Vegetación}

En los 28 sitios caracterizamos la estructura de la vegetación. Para ello se registró la altura modal y la cobertura de las principales formas de crecimiento. Las formas de crecimiento consideradas fueron paja fina (incluye especies de crecimiento en mata de hojas finas como 
Deyeuxia hieronymi (Hack.) Türpe y otras), paja gruesa (incluye a las especies de crecimiento en mata de hojas gruesas, en los sitios de estudio solamente Poa stuckertii (Hack.) Parodi), graminoides (perennes y anuales como Carex spp., Bromus spp., entre otras), hierbas bajas (Lachemilla pinnata (Ruiz \& Pav.) Rothm., Eryngium nudicaule Lam., entre otras), árboles ( $P$. australis y Maytenus boaria Molina), arbustos (incluye arbustos y sufrútices como Berberis hieronymi C.K. Schneid., Gaultheria poeppigii DC., Stevia spp., entre otras), helechos (Blechnum penna-marina (Poir.) Kuhn, entre otros), cactus (Gymnocalycium spp.), líquenes y musgos. También estimamos visualmente la cobertura (\%) de broza, suelo desnudo, roca expuesta (pedregullo y pavimentos resultado de la erosión del suelo) y de afloramientos rocosos en el total de cada uno de los sitios. Estos últimos no son el resultado de la erosión reciente del suelo sino de procesos geomorfológicos históricos, y se diferencian de los pavimentos de erosión por su mayor altura sobre el nivel del suelo, por estar cubiertos con mayor proporción de líquenes y por poseer numerosas fisuras donde crecen algunas plantas (Cingolani et al. 2013). Cabe aclarar que la cobertura total de los sitios en algunos casos sumó más de $100 \%$, ya que las canopias de las distintas formas de crecimiento se superponían. A partir de la cobertura y altura modal de las formas de crecimiento calculamos un índice de estructura de la vegetación (Ecuación 1).

Índice de estructura $\mathrm{j}_{\mathrm{j}}=\sum\left(\mathrm{a}_{\mathrm{ij}}{ }^{*} \mathrm{c}_{\mathrm{ij}}\right) / 100$

Donde $\mathrm{a}_{\mathrm{ij}}$ es la altura modal $(\mathrm{cm})$ de la forma de crecimiento i en el sitio j y $c_{i j}$ la cobertura (\%) de la forma de crecimiento i en el sitio j. Por sus características, asumimos que este índice refleja indirectamente la biomasa, ya que aumenta a medida que también lo hacen la altura, la cobertura y la superposición de estratos de la vegetación. En diversos estudios se han utilizado variables relacionadas a la altura y la cobertura de la vegetación para estimar la biomasa (e.g., Conti et al. 2013; Poca et al. 2015).

También medimos el índice de área foliar (i.e., la relación área de hojas/área de terreno) de cada sitio utilizando un LAI 2000 Li-Cor con un anillo de $90^{\circ}$. Para la densidad de raíces $\left(\mathrm{g} / \mathrm{cm}^{3}\right)$ de los primeros $15 \mathrm{~cm}$ de suelo, en un punto al azar por sitio tomamos muestras de suelo con un cilindro metálico de $161 \mathrm{~cm}^{3}$, las tamizamos con un tamiz de $2 \mathrm{~mm}$ de malla (recuperando las raíces), las secamos en estufa a $60{ }^{\circ} \mathrm{C}$ durante $48 \mathrm{~h}$ y las pesamos con una balanza de precisión ( $\pm 1 \mathrm{mg})$.

Adicionalmente, realizamos censos florísticos completos en cada sitio. Para ello estimamos visualmente la cobertura de la totalidad de especies de plantas vasculares presentes en los sitios en categorías de 5\% (10\%, 15\%, 20\%, etc.), excepto para valores bajos de cobertura $(<10 \%)$, que estimamos en categorías de $1 \%$ (1\%, $2 \%$, etc.). Cuando la cobertura fue menor al $1 \%$ registramos $0.1 \%$.

Por último, y sólo con fines ilustrativos, para facilitar la interpretación de los gráficos presentados en resultados, clasificamos de forma visual y cualitativa los sitios según la forma de vida dominante en los siguientes tipos fisonómicos: bosques, matorrales, pajonales, céspedes, helechales y pedregales.

\section{Suelo}

En cada uno de los 28 sitios medimos en 10 puntos al azar la compactación del suelo $(\mathrm{kg} /$ $\mathrm{cm}^{2}$ ) con un penetrómetro de bolsillo (Forestry Suppliers Inc.), evitando las zonas rocosas. A su vez, medimos la densidad aparente de los primeros $15 \mathrm{~cm}$ del suelo en un punto al azar por sitio, tomando muestras con un cilindro metálico de volumen conocido $\left(161 \mathrm{~cm}^{3}\right)$. Tamizamos las muestras con un tamiz de 5 $\mathrm{mm}$ de malla para separar las rocas. Medimos el volumen de las rocas con una probeta para descontarlo del volumen total de la muestra. Secamos el suelo en estufa a $105^{\circ} \mathrm{C}$ durante 24 horas para eliminar toda la humedad, y luego lo pesamos. Calculamos la densidad aparente dividiendo el peso por el volumen de suelo $\left(\mathrm{g} / \mathrm{cm}^{3}\right)$ (Throop et al. 2012).

Por otra parte, extrajimos cinco muestras de suelo al azar por sitio de los primeros $15 \mathrm{~cm}$ de profundidad, que se mezclaron para obtener una muestra compuesta representativa de cada sitio. Dichas muestras se trasladaron al Laboratorio de Edafología de la Facultad de Ciencias Agropecuarias de la Universidad Nacional de Córdoba, donde se secaron en estufa de circulación forzada. Luego, se molieron y tamizaron con una malla de $2 \mathrm{~mm}$ para su posterior análisis físico y químico. Las variables que se determinaron fueron contenido de arena, limo y arcilla (\%), $\mathrm{pH}$, conductividad eléctrica $(\mathrm{dS} / \mathrm{m})$, materia orgánica $(\%)$, carbono orgánico (\%) mediante la técnica Walkley-Black (Nelson and Sommers 1996), nitrógeno total (\%) mediante la técnica Kjeldahl (Bremner 1996), relación C:N, 
fósforo extractable (ppm) mediante la técnica Bray y Kurtz №1, capacidad de intercambio catiónico (meq/100 g), cationes $\left(\mathrm{Na}^{+}, \mathrm{Ca}^{2+}, \mathrm{Mg}^{2+}\right.$ $\mathrm{y} \mathrm{K}^{+}$, meq/100 $\left.\mathrm{g}\right)$, sodio intercambiable (\%), capacidad de campo (\%), punto de marchitez permanente (\%) y el agua disponible para las plantas o agua útil (\%). Para las mediciones de capacidad de campo y punto de marchitez permanente, a la muestra de suelo se la pesa al inicio y luego se le aplica una presión de -0.3 MPa y luego de -1.5 MPa. Después de aplicar cada presión se pesa la muestra de suelo para registrar la diferencia de peso respecto al inicial y así obtener el contenido de agua a cada una de las presiones ejercidas. El valor correspondiente a la presión de $0.3 \mathrm{MPa}$ equivale a la capacidad de campo, mientras que aquél a la presión de -1.5 MPa equivale al punto de marchitez permanente (Kirkham 2005). El agua útil se calculó a partir de la diferencia de la capacidad de campo y el punto de marchitez permanente.

Como indicadores de la capacidad de almacenamiento de agua en el suelo, en 7 a 10 puntos al azar por sitio medimos la profundidad del suelo con una varilla de acero de $1 \mathrm{~m}$ de longitud, y evitamos las zonas de roca expuesta. Consideramos como profundidad del suelo la sección de la varilla que quedaba enterrada luego de clavarla con repetidos golpes de masa hasta que dejara de hundirse por resistencia de la roca madre. En el caso que la varilla se enterrara casi por completo $(95 \mathrm{~cm})$ sin tocar la roca madre lo registramos como $100 \mathrm{~cm}$. Promediamos los valores de profundidad del suelo para obtener un valor único representativo por sitio. Sólo dos sitios presentaron un valor promedio de $100 \mathrm{~cm}$. Finalmente, medimos el tiempo de infiltración (s), que es una medida de cuán rápido penetra el agua en el suelo. Para ello utilizamos un anillo metálico de $10 \mathrm{~cm}$ de diámetro que se enterró a $5 \mathrm{~cm}$ de profundidad en un punto al azar por sitio con cuidado para evitar disturbar el suelo. Previamente, cortamos la vegetación al ras del suelo. Vertimos $250 \mathrm{ml}$ de agua y registramos el tiempo que tardó el agua en penetrar el suelo (técnica modificada de USDA 1999). A partir del tiempo de infiltración (s) registrado, calculamos para cada sitio una tasa de infiltración $(\mathrm{cm} / \mathrm{h})$ siguiendo la Ecuación 2 propuesta originalmente por Bouwer (1986).

Tasa de infiltración $(\mathrm{cm} / \mathrm{h})=\mathrm{Q} /\left(\mathrm{A} \times \mathrm{T}_{\mathrm{j}}\right)$

donde $Q$ es cantidad de agua $\left(250 \mathrm{~cm}^{3}\right)$, A el área del cilindro $\left(78,5 \mathrm{~cm}^{2}\right)$ y $\mathrm{T}$ el tiempo de infiltración del sitio $\mathrm{j}(\mathrm{h})$.

\section{Análisis de datos}

Para cumplir con el primer objetivo específico utilizamos métodos multivariados y correlaciones. Por un lado, realizamos un análisis de componentes principales (CP) con las seis variables topográficas obtenidas para los 28 sitios. Este análisis permitió observar la posición relativa de los sitios en relación a dichas variables en conjunto $y$, a su vez, resumirlas en dos ejes principales (CP 1 y CP 2). Correlacionamos a través de Pearson estos dos primeros ejes con las seis variables y seleccionamos la variable que mejor se correlacionó con el eje 1 y la que mejor se correlacionó con el eje 2, como representativas de la variabilidad topográfica.

Por otro lado, utilizamos el índice de estructura, la densidad de raíces y el índice de área foliar como variables descriptoras de la vegetación. Además, resumimos la composición florística de los 28 sitios de estudio a través de un análisis de correspondencia libre de tendencia (DCA por sus siglas en inglés) a partir de una matriz de censos por especies (28x119). El total de especies registradas fue 149, pero para el análisis se descartaron 30 especies raras que sólo estaban presentes en uno o dos sitios. Seleccionamos al eje 1 (DCA 1) como la variable resumen de la composición florística. Finalmente, correlacionamos las cuatro variables descriptoras de las características de la vegetación (índice de estructura, densidad de raíces, el índice de área foliar y DCA 1) entre sí.

Para resumir la variación en las características físico-químicas de la capa superficial del suelo realizamos otro análisis de $\mathrm{CP}$, para lo cual utilizamos 19 de las 21 variables edáficas de los primeros $15 \mathrm{~cm}$ del suelo. En el análisis de CP no incluimos la compactación ni la densidad aparente porque debido a su potencial importancia en la tasa de infiltración nos interesaba considerarlas individualmente. Tampoco incluimos la tasa de infiltración y la profundidad del suelo, debido a que teníamos por objetivo analizar como las restantes variables de suelo se relacionaban con estas dos variables, que son indicadoras de la capacidad de almacenamiento de agua (tercer objetivo específico). A través de correlaciones de Pearson seleccionamos las dos variables que mejor se correlacionaron con el CP 1 y las dos que mejor se correlacionaron con el CP 2, como representativas de la variabilidad edáfica. Finalmente, correlacionamos la 
densidad aparente y compactación con las cuatro variables seleccionadas a partir del análisis de CP.

Para alcanzar el segundo objetivo específico, analizamos el nivel de asociación entre las cuatro variables descriptoras de la vegetación, las seis variables edáficas superficiales y las dos variables topográficas. Para cumplir con el tercer objetivo específico realizamos correlaciones de Pearson de las dos variables indicadoras de la capacidad del suelo de almacenar agua (tasa de infiltración y profundidad del suelo) con las cuatro variables de la vegetación, las seis variables edáficas superficiales y las dos variables topográficas. En todos los casos utilizamos el programa estadístico InfoStat versión 2013 (Di Rienzo et al. 2013), excepto para el DCA que utilizamos el programa PC-ORD versión 6.0 (McCune and Mefford 1999).

\section{Resultados}

\section{Topografía}

El CP 1 del análisis multivariado realizado a partir de las variables topográficas explicó el $35.6 \%$ de la varianza; el CP 2, el $20.0 \%$ (Figura 1). Los sitios más altos en el paisaje y con menor pendiente se ubican hacia el extremo positivo del CP 1, mientras que los sitios bajos, con mayor pendiente y más rugosos se ubican en el extremo negativo de dicho eje. A lo largo del CP 2, los sitios con mayor insolación anual y área de acumulación se ubican hacia el extremo positivo y los sitios con las características opuestas se ubican en el extremo negativo (Figura $1 \mathrm{a} \mathrm{v}$ b). No se observa una clara agrupación de los tipos fisonómicos en el espacio definido por los dos ejes (Figura 1a). La variable topográfica más representativa de cada uno de los dos primeros ejes del análisis de CP y sus rangos de variación entre sitios fueron: para el CP 1 , altitud (2040 - 2100 m s.n.m; r=0.81, P<0.001) y para el CP 2, insolación anual (-19-18; r=0.86, $P<0.001)$.

\section{Vegetación}

El índice de estructura de la vegetación varió entre 1.1 y 392.8, el índice de área foliar entre 0.08 y 4.6 y la densidad de raíces entre 0.001 y $0.03 \mathrm{~g} / \mathrm{cm}^{3}$ para los 28 sitios de estudio. Los dos primeros ejes del DCA florístico presentaron autovalores de 0.80 y 0.36 , respectivamente (Figura 2). Hacia el extremo positivo del DCA 1 se ubican los pedregales caracterizados por F. tucumanica, Plantago brasiliensis Sims, Nassella nidulans (Mez) Barkworth y céspedes cuyas especies dominantes son C. fuscula, $L$. pinnata y Eryngium nudicaule Lam. Hacia el centro de ambos ejes del DCA se ubicaron los pajonales, dominados por $D$. hieronymi principalmente (Figura 2a y b). Los restantes sitios se ubicaron en el extremo negativo del DCA 1 y se diferenciaron entre sí a lo largo del DCA 2. Este segundo eje estuvo determinado principalmente por la cobertura de $P$. australis hacia el extremo negativo, lo cual caracterizó a los bosques. En el centro de dicho eje se ubican los helechales caracterizados por $B$. penna-marina y en el extremo positivo se ubican los matorrales, caracterizados por Clinopodium spp., B. hieronymi y G. poepiggii (Figura 2b). El índice de estructura de la vegetación se asoció marginal y positivamente al índice de


Figura 1. a) Distribución de los 28 sitios en los dos primeros ejes del análisis de componentes principales (CP) basado en las variables topográficas. Los símbolos indican la fisonomía definida a priori: $(\bullet)$ bosque, $(\mathbf{\Lambda})$ matorral, $(\Delta)$ pajonal, (O) césped, ( $)$ helechal y $(\square)$ pedregal. b) Contribución de las variables topográficas al ordenamiento.

Figure 1. a) Distribution of the 28 sites in the first two axis of the principal component (CP) analysis based on the topographic variables. Symbols indicate the physiognomy defined a priori: $(\bullet)$ woodland, $(\boldsymbol{\Delta})$ shrubland, $(\Delta)$ tussock grassland, (O) lawn, ( $\bullet$ ) fernland and (๑) stoneland. b) Contribution of topographic variables to the ordination. 
área foliar $(\mathrm{r}=0.36, P=0.06)$ y negativamente al DCA 1 ( $r=-0.61, P<0.001)$. Sin embargo, no se asoció a la densidad de raíces $(\mathrm{r}=-0.03, P=0.90)$. Por lo tanto, de aquí en adelante se presentan los resultados sólo para índice de estructura, índice de área foliar y densidad de raíces.

\section{Suelo}

La densidad aparente varió entre 0.28 a 1.55 $\mathrm{g} / \mathrm{cm}^{3}$, mientras que la compactación tuvo valores entre 0.59 y $3.72 \mathrm{~kg} / \mathrm{cm}^{2}$ en los 28 sitios. Debido a la estrecha correlación entre estas dos variables $(r=0.53, P<0.01)$, de aquí en adelante se presentan sólo los resultados para la densidad aparente. La variación de las restantes características edáficas de los primeros $15 \mathrm{~cm}$ del perfil de suelo se resumen en el análisis multivariado de la Figura 3, donde el CP 1 explicó el 37.6\% de la varianza y el CP 2 el 16.3\%. El extremo positivo del
CP 1 se caracteriza por suelos con un mayor contenido de materia orgánica, nitrógeno total y carbono orgánico, así como altos valores de las constantes hídricas (capacidad de campo, punto de marchitez permanente y agua disponible). En cambio, los suelos de los sitios ubicados en el extremo negativo del CP 1 presentan los atributos opuestos $\mathrm{y}$ un alto porcentaje de fósforo extractable (Figura 3a y b). El CP 2 estuvo determinado principalmente por la textura. Los suelos de los sitios ubicados hacia el extremo positivo presentaron mayor porcentaje de arena en relación a los del extremo opuesto que tienen un mayor porcentaje de arcilla y limo (Figura 3a y b). En general, hay una tendencia de sitios con vegetación moderadamente a muy estructurada (helechales, pajonales, matorrales y bosques) a ubicarse en el extremo positivo del CP 1 (Figura 3a). Las dos variables edáficas
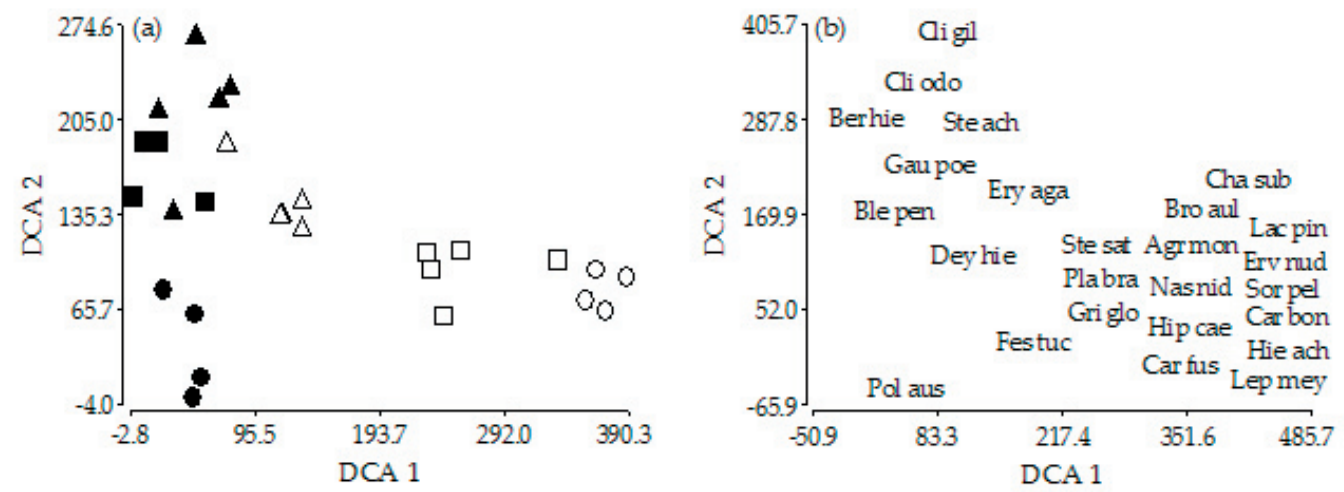

Figura 2. a) Distribución de los 28 sitios en los dos primeros ejes del análisis de correspondencia libre de tendencia (DCA) basado en la identidad y abundancia de las especies de plantas vasculares. Los símbolos indican la fisonomía definida a priori: $(\bullet)$ bosque, $(\boldsymbol{\Lambda})$ matorral, $(\Delta)$ pajonal, $(\mathrm{O})$ césped, $(\bullet)$ helechal y $(\square)$ pedregal. b) Contribución de las especies más representativas al ordenamiento. Se indica con las tres primeras letras el nombre del género y la especie tal como se detalla a continuación. Pol aus: Polylepis australis Bitter; Ber hie: Berberis hieronymi C.K. Schneid.; Cli odo: Clinopodium odorum (Griseb.) Harley; Cli gil: Clinopodium gilliesii (Benth.) Kuntze; Gau poe: Gaultheria poeppigii DC.; Ste ach: Stevia achalensis Hieron.; Ste sat: Stevia satureiifolia (Lam.) Sch. Bip. Ery aga: Eryngium agavifolium Griseb.; Ery nud: Eryngium nudicaule Lam.; Gri glo: Grindelia globularifolia Griseb.; Hie ach: Hieracium achalense Sleumer; Hyp cae: Hypochaeris caespitosa Cabrera; Lac pin: Lachemilla pinnata (Ruiz \& Pav.) Rothm.; Lep mey: Lepechinia meyenii (Walp.) Epling; Pla bra: Plantago brasiliensis Sims; Dey hie: Deyeuxia hieronymi (Hack.) Türpe; Fes tuc: Festuca tucumanica E. B. Alexeev; Agr mon: Agrostis montevidensis Spreng. ex Nees; Bro aul: Bromus auleticus Trin. ex Nees; Car bon: Carex bonariense Desf. ex Poir.; Car fus: Carex fuscula d'Urv.; Cha sub: Chascolytrum subaristatum (Lam.) Desv.; Jar jun: Jarava juncoides (Speg.) Peñailillo; Nas nid: Nassella nidulans (Mez) Barkworth; Sor pel: Sorghastrum pellitum (Hack.) Parodi; Ble pen: Blechnum penna-marina (Poir.) Kuhn.

Figure 2. a) Distribution of the 28 sites in the first two axis of the detrended correspondence analysis (DCA) based on the identity and abundance of plant vascular species. Symbols indicate the physiognomy defined a priori: $(\bullet)$

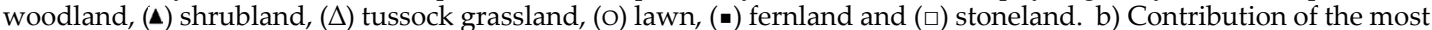
relevant species to the ordination. Genus and species names are indicated with the first three letters as follow. Pol aus: Polylepis australis Bitter; Ber hie: Berberis hieronymi C.K. Schneid.; Cli odo: Clinopodium odorum (Griseb.) Harley; Cli gil: Clinopodium gilliesii (Benth.) Kuntze; Gau poe: Gaultheria poeppigii DC.; Ste ach: Stevia achalensis Hieron.; Ste sat: Stevia satureiifolia (Lam.) Sch. Bip. Ery aga: Eryngium agavifolium Griseb.; Ery nud: Eryngium nudicaule Lam.; Gri glo: Grindelia globularifolia Griseb.; Hie ach: Hieracium achalense Sleumer; Hyp cae: Hypochaeris caespitosa Cabrera; Lac pin: Lachemilla pinnata (Ruiz \& Pav.) Rothm.; Lep mey: Lepechinia meyenii (Walp.) Epling; Pla bra: Plantago brasiliensis Sims; Dey hie: Deyeuxia hieronymi (Hack.) Türpe; Fes tuc: Festuca tucumanica E. B. Alexeev; Agr mon: Agrostis montevidensis Spreng. ex Nees; Bro aul: Bromus auleticus Trin. ex Nees; Car bon: Carex bonariense Desf. ex Poir.; Car fus: Carex fuscula d'Urv.; Cha sub: Chascolytrum subaristatum (Lam.) Desv.; Jar jun: Jarava juncoides (Speg.) Peñailillo; Nas nid: Nassella nidulans (Mez) Barkworth; Sor pel: Sorghastrum pellitum (Hack.) Parodi; Ble pen: Blechnum penna-marina (Poir.) Kuhn. 

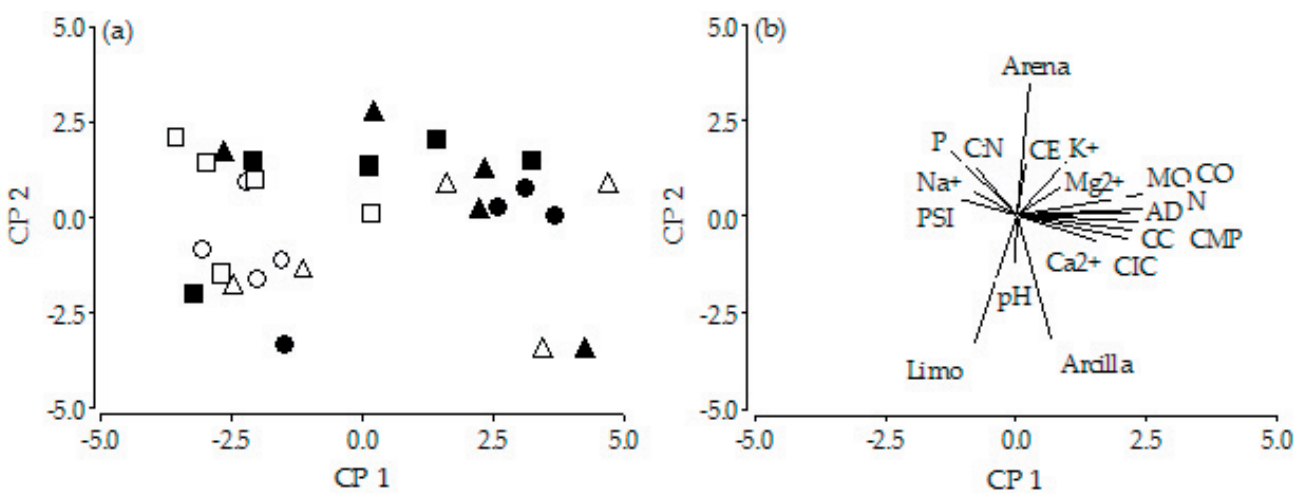

Figura 3. a) Distribución de los 28 sitios en los dos primeros ejes del análisis de componentes principales (CP) basado en las variables edáficas de los primeros $15 \mathrm{~cm}$ del perfil de suelo. Los símbolos indican la fisonomía definida a priori: $(\bullet)$ bosque, $(\boldsymbol{\Delta})$ matorral, $(\Delta)$ pajonal, $(\mathrm{O})$ césped, $(\bullet)$ helechal y $(\square)$ pedregal. b) Contribución de las variables edáficas al ordenamiento. Las abreviaturas indican: P: fósforo, C:N: relación carbono:nitrógeno, Na+: sodio, PSI: porcentaje de sodio intercambiable, $\mathrm{CE}$ : conductividad eléctrica, $\mathrm{K}+$ : potasio, $\mathrm{Mg} 2+$ : magnesio, $\mathrm{N}$ : nitrógeno, $\mathrm{Ca} 2+$ : calcio $\mathrm{CE}$ : conductividad eléctrica, CO: carbono orgánico, MO: materia orgánica, CIC: capacidad de intercambio catiónico, AD: agua disponible para las plantas, CC: capacidad de campo, PMP: punto de marchitez permanente.

Figure 3. a) Distribution of the 28 sites in the first two axis of the principal component $(\mathrm{CP})$ analysis following edaphic variables of the first $15 \mathrm{~cm}$ of the soil profile. Symbols indicate the physiognomy defined a priori $(\bullet)$ woodland, $(\mathbf{\Delta})$ shrubland, $(\Delta)$ tussock grassland, (O) lawn, (- ) fernland and ( $\square$ ) stoneland. b) Contribution of edaphic variables to the ordination. Abbreviations indicate: P: phosphorous, C:N: carbon:nitrogen relation, Na+: sodium, PSI: percentage of exchangeable sodium, $\mathrm{CE}$ : electric conductivity, $\mathrm{K+}$ : potassium, $\mathrm{Mg} 2+$ : magnesium, $\mathrm{N}$ : nitrogen, $\mathrm{Ca} 2+$ : calcium, CO: organic carbon, MO: organic matter, CIC: cationic exchangeable capacity, AD: plant available water, CC: field capacity, PMP: wilting permanent point.

más representativas de cada uno de los dos primeros ejes de los análisis de CP y sus rangos de variación entre sitios fueron: para el CP 1, materia orgánica (5.4-13.3\%; r=0.96, $P<0.001)$ y capacidad de campo $(15.7-39.1 \%$ ; $\mathrm{r}=0.92, P<0.001$ ); para el CP 2, arena (67.3$82 \%$; $r=0.93, P<0.001)$ y limo $(12.6-23.6 \%$; $\mathrm{r}=0.85, P<0.001)$. La densidad aparente se asoció estrecha y negativamente al contenido de materia orgánica $(\mathrm{r}=-0.61, P<0.001)$ y a la capacidad de campo $(\mathrm{r}=-0.75, P<0.01)$, y positiva y marginalmente al contenido de limo ( $\mathrm{r}=0.37, P=0.06)$; sin embargo, no se relacionó significativamente con el contenido de arena $(\mathrm{r}=-0.17, P=0.38)$.

\section{Relación vegetación, topografía y suelo}

Las tres variables descriptoras de la vegetación se asociaron negativamente a la densidad aparente $(\mathrm{r}=-0.53, P<0.01$ para el índice de estructura de la vegetación; $\mathrm{r}=-0.39, P<0.05$ para el índice de área foliar; $\mathrm{r}=0.61, P<0.001$ para la densidad de raíces). Además, aquellos sitios con vegetación más estructurada presentaron suelos con mayor contenido de materia orgánica $(\mathrm{r}=0.43, P<0.05)$ y capacidad de campo $(\mathrm{r}=0.46, P<0.01)$. En general, no detectamos asociaciones entre las variables topográficas y las relacionadas a la vegetación y el suelo (Tabla A del Apéndice). Las excepciones a ello fueron la relación

Tabla 1. Coeficientes de correlación de Pearson de la tasa de infiltración y la profundidad del suelo con las variables topográficas y variables edáficas más relevantes seleccionadas a partir de los análisis de componentes principales para los 28 sitios de estudio.

Table 1. Pearson correlation coefficients of infiltration rate and soil depth with the vegetation structure index, and the most relevant topographic and edaphic variables selected from the principal component analysis for the 28 sites.

\begin{tabular}{|c|c|c|c|c|}
\hline \multirow[b]{2}{*}{ Índice de estructura de la vegetación } & \multicolumn{2}{|c|}{ Tasa de infiltración $(\mathrm{cm} / \mathrm{h})$} & \multicolumn{2}{|c|}{ Profundidad del suelo $(\mathrm{cm})$} \\
\hline & 0.35 & & 0.55 & ** \\
\hline Índice de área foliar & 0.17 & & 0.35 & \\
\hline Densidad de raíces $\left(\mathrm{g} / \mathrm{cm}^{3}\right)$ & 0.38 & * & 0.10 & \\
\hline Densidad aparente $\left(\mathrm{g} / \mathrm{cm}^{3}\right)$ & -0.59 & ** & -0.65 & $* * *$ \\
\hline Altitud (m s.n.m.) & -0.17 & & -0.14 & \\
\hline Índice de insolación anual $\left({ }^{\circ}\right)$ & -0.03 & & -0.16 & \\
\hline Materia organica $(\%)$ & 0.41 & * & 0.54 & $* *$ \\
\hline Capacidad de campo (\%) & 0.48 & $* *$ & 0.58 & $* * *$ \\
\hline Arena $(\%)$ & 0.39 & * & 0.02 & \\
\hline Limo (\%) & -0.52 & ** & -0.15 & \\
\hline
\end{tabular}



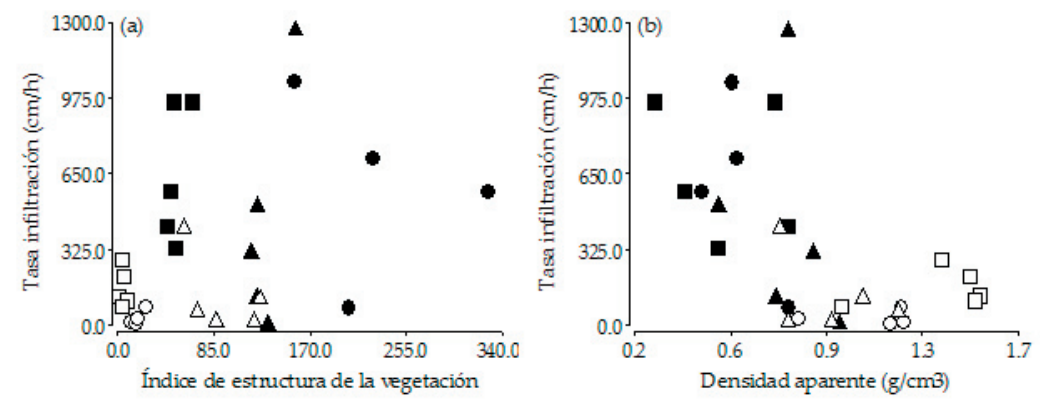

Figura 4. Diagramas de dispersión entre la tasa de infiltración del suelo $(\mathrm{cm} / \mathrm{h})$ y: a) el índice de estructura de la vegetación, b) la densidad aparente $\left.\left(\mathrm{g} / \mathrm{cm}^{3}\right), \mathrm{c}\right)$ la capacidad de campo (\%), y d) el contenido de materia orgánica (\%). Los símbolos indican la fisonomía: $(\bullet)$ bosque, $(\boldsymbol{\Lambda})$ matorral, $(\Delta)$ pajonal, (O)
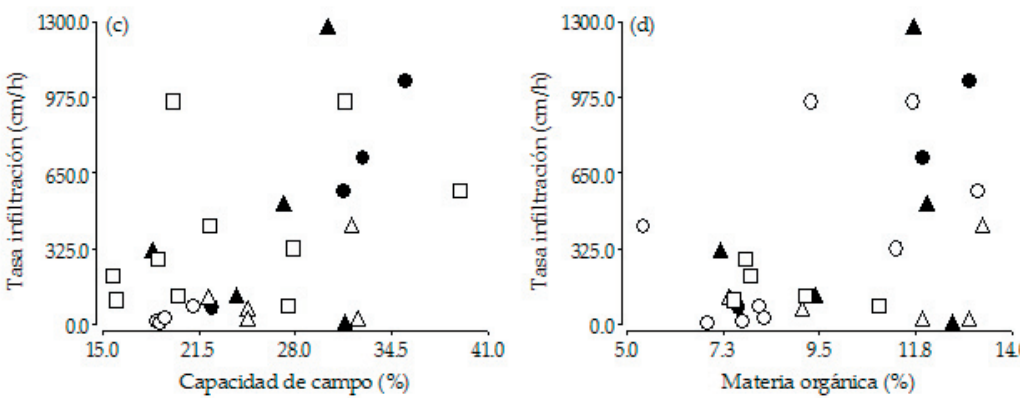
césped, (•) helechal y ( $\square$ ) pedregal.

Figure 4. Scatter plot between infiltration rate $(\mathrm{cm} / \mathrm{h})$ and: a) vegetation structure index, b) soil bulk density $\left(\mathrm{g} / \mathrm{cm}^{3}\right)$ c) field capacity (\%), and d) organic matter content (\%). Symbols indicate the physiognomy: $(\bullet)$ woodland, $(\Delta)$ shrubland, $(\Delta)$ tussock grassland, (O) lawn, (- )
fernland and ( $\square$ ) stoneland.
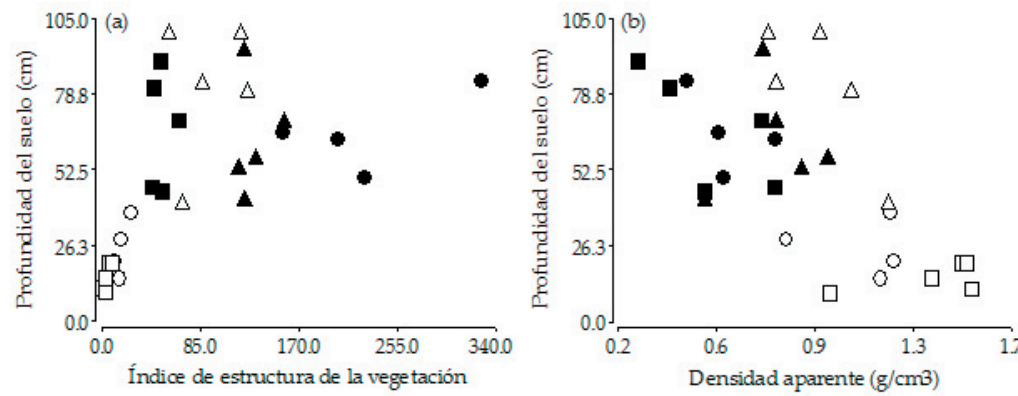

Figura 5. Diagramas de dispersión entrela profundidad del suelo (cm) y: a) el índice de estructura de la vegetación, b) la densidad aparente $\left.\left(\mathrm{g} / \mathrm{cm}^{3}\right), \mathrm{c}\right)$ la capacidad de campo (\%), y d) el contenido de materia orgánica (\%). Los símbolos indican la fisonomía: $(\bullet)$ bosque, $(\boldsymbol{\Lambda})$ matorral, $(\Delta)$
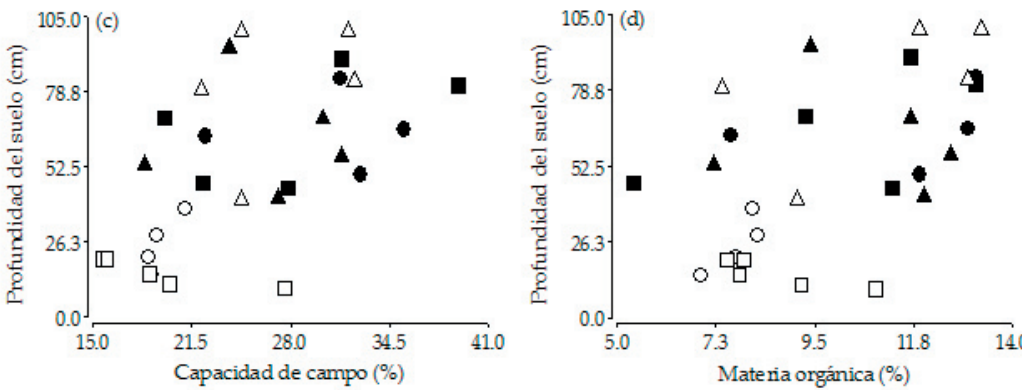
pajonal, (O) césped, (-) helechal y (口) pedregal.

Figure 5. Scatter plot between soil depth (cm) and: a) vegetation structure index, b) soil bulk density $\left(\mathrm{g} / \mathrm{cm}^{3}\right)$, c) field capacity (\%), and d) organic matter content (\%). Symbols indicate the physiognomy: $(\bullet)$ woodland, $(\Delta)$ shrubland, $(\Delta)$ tussock grassland, (O) lawn, (-)

negativa del índice de insolación anual con la densidad de raíces y la relación positiva de la altitud con la densidad aparente, en ambos casos relaciones débiles. Sin embargo, ni la altitud ni la insolación anual se asociaron a la estructura de la vegetación (Tabla A del Apéndice).

\section{Capacidad de almacenamiento de agua}

La tasa de infiltración varió entre 7.06 y $1273.9 \mathrm{~cm} / \mathrm{h}$, y la profundidad del suelo entre 9.6 y $100 \mathrm{~cm}$. Los sitios con mayor tasa de infiltración tendieron a presentar suelos más profundos $(r=0.36, P=0.06)$. Tanto la tasa de infiltración como la profundidad del suelo fueron mayores en sitios con vegetación más estructurada, cuyos suelos son menos densos y tienen mayor capacidad de campo y contenido de materia orgánica (Tabla 1 y Figura 4a-d para la tasa de infiltración; Figura 5a-d para la profundidad del suelo). Además, la tasa de infiltración se vio favorecida en sitios con mayor densidad de raíces y suelos con mayor contenido de arena y menor contenido de limo (Tabla 1). 


\section{DisCUSIÓN}

En este trabajo encontramos que los cambios en la estructura de la vegetación en los bosques de altura de las sierras de Córdoba, principalmente a causa de la historia de uso, se asociaron también a cambios en las propiedades edáficas, que comprenden alteraciones en la tasa de infiltración y en la profundidad del suelo. De estas dos variables depende, principalmente, la capacidad de almacenamiento de agua del suelo (NoyMeyr 1973). Por lo tanto, nuestros resultados indican que mayor cantidad de agua es capaz de ingresar al suelo y ser almacenada mientras más conservado se encuentre el sistema vegetación-suelo de los bosques de $P$. australis. Esto incluye que la vegetación tenga mayor complejidad estructural y los suelos presenten mayor contenido de materia orgánica y capacidad de campo, así como menor densidad aparente.

Ninguna de las variables topográficas se relacionó significativamente a la estructura de la vegetación ni a las variables indicadoras de almacenamiento de agua en el suelo. Es decir, podemos considerar que las variaciones en el sistema vegetación-suelo encontradas se deben principalmente al disturbio histórico y reciente sin una interferencia importante de la topografía. Sin embargo, no podemos descartar que haya otros efectos no contemplados en este trabajo (como puede ser la microtopografía [o microrugosidad] de la roca madre) que también influyan sobre las variables edáficas y de vegetación.

Además de la complejidad estructural dada por la superposición de estratos, el índice de estructura de la vegetación reflejó el recambio en la composición florística y, marginalmente, el área foliar de los sitios. Sin embargo, el índice de estructura no se asoció a la densidad de raíces, ya que la mayor densidad se encontró en sitios con índice de estructura intermedio, dominados por helechos. Es posible que la profundidad muestreada $(15 \mathrm{~cm})$ no haya sido suficiente para captar de forma representativa la densidad de raíces de los sitios con mayor complejidad estructural, los bosques. Es conocido que las especies leñosas distribuyen sus raíces a lo largo del perfil del suelo, y alcanzan profundidades mayores a $15 \mathrm{~cm}$. Lo mismo podría estar sucediendo en los sitios dominados por pajonales (Canadell et al. 1996). De hecho, es común observar que las raíces de los árboles de $P$. australis alcanzan profundidades mayores a las $50 \mathrm{~cm}$ (observación personal), mientras que las raíces de los céspedes, en particular, disminuyen con la profundidad sin superar los $30 \mathrm{~cm}$ (Vaieretti et al. en preparación).

Los sitios con vegetación menos estructurada tuvieron suelos menos profundos y con mayor densidad aparente, lo que sugiere que el disturbio histórico y actual, que transformó la estructura de la vegetación, también produjo la pérdida y la compactación de los suelos. A nivel global, diversos trabajos han señalado el impacto negativo del pastoreo y el fuego sobre la densidad aparente y la compactación del suelo (Boone Kauffman et al. 2004; YongZhong et al. 2005; Altesor et al. 2006). Para los ecosistemas de altura de las sierras de Córdoba se ha detectado que los sitios con vegetación menos estructurada tienen una carga ganadera puntual mayor (von Müller et al. 2017). Además, los sitios con vegetación más estructurada se ubicaron principalmente en el establecimiento sin ganado (Figura $1 b$ y c del Apéndice Electrónico) debido a que en los otros dos establecimientos resultaba muy difícil encontrar este tipo de parche. Este potrero no tiene animales desde hace 20 años, a partir de la creación del Parque Nacional Quebrada del Condorito. A su vez, hay indicios de que el Parque tuvo el menor impacto histórico de ambos disturbios (Cingolani et al. 2004, 2008). Adicionalmente, los sitios con vegetación menos estructurada presentaron menor contenido de materia orgánica y capacidad de campo, que, a su vez, tienen menor contenido de nitrógeno y carbono orgánico, así como menor cantidad de agua disponible para las plantas. Numerosos trabajos indicaron que a causa de la reducción en la cobertura de la canopia $\mathrm{y}$, consecuentemente, del aporte de broza, el ganado destruye la estructura de la parte superficial del suelo y aumenta la densidad aparente y la compactación del mismo, y disminuye la cantidad de materia orgánica y la capacidad de retención de agua del suelo (Boone Kauffman et al. 2004; Yong-Zhong et al. 2005; Altesor et al. 2006). Por el contrario, la vegetación estructurada y moderadamente estructurada de bosques, matorrales y pajonales previno la erosión del suelo, ya que presentaron suelos con los atributos opuestos, probablemente también debido al mayor aporte de broza de la vegetación. Esto se ve reflejado en la mayor acumulación de materia orgánica, nitrógeno y un aumento en las constantes hídricas.

Se ha propuesto que el mecanismo por el cual sitios con vegetación más estructurada presentan una tasa de infiltración más rápida es que hay un mayor aporte de broza al suelo, lo 
que aumenta la cantidad de materia orgánica. De este modo, la estructura del suelo se ve favorecida, ya que es menor la compactación y la densidad aparente (Neary et al. 2009; Bonell et al. 2010; Germer et al. 2010). En consecuencia, se incrementa la proliferación de raíces y la aireación del suelo; esto, en conjunto, favorece la tasa de infiltración (Throop et al. 2012). En coincidencia con dichos trabajos, encontramos que los sitios con vegetación estructurada y/o alta densidad de raíces, además de presentar dichas propiedades del suelo, tuvieron una mayor tasa de infiltración. La textura del suelo es normalmente otra de las variables determinantes de la tasa de infiltración. Suelos con altos contenidos de arena suelen tener mayores oportunidades de infiltración y percolación, aunque baja retención de humedad (Gómez-Plaza et al. 2001). En coincidencia con los autores, encontramos que la proporción de arena y limo fueron relevantes para la tasa de infiltración, a pesar de que la textura del suelo, en términos generales, fue similar entre los sitios; varió de arenosa-franco a franco-arenosa.

El impacto negativo del pastoreo y del fuego sobre la erosión del suelo es conocido (Pimentel et al. 1995; Wondzell and King 2003). Para el área de estudio, Cingolani et al. (2013) detectaron que se pierden por año 0.6 $\mathrm{cm}$ de suelo en sitios quemados y pastoreados, mientras que esta tasa disminuye a $0.4 \mathrm{~cm}$ si se excluye el ganado luego de un incendio. Nuestros resultados, abarcando la variabilidad completa en la estructura de la vegetación, indican no sólo la asociación negativa entre la profundidad y la densidad aparente del suelo, sino también que sitios con vegetación menos estructurada presentan suelos menos profundos. Pudimos detectar una diferencia en la profundidad del suelo tan extrema como de 100 a $9 \mathrm{~cm}$ entre un bosque y un pedregal, en el último caso considerando las porciones de suelo remanente en dichos sitios. La falta de vegetación que produce un incendio o el consumo de los herbívoros promueve no sólo la erosión del suelo; también dificulta la infiltración del agua y, con ello, el crecimiento de la vegetación y la recuperación del sistema. Se genera, por lo tanto, una retroalimentación positiva entre la falta de vegetación y la erosión de los suelos. Es decir, cuanto más se erosiona un suelo, más difícil resulta el crecimiento vegetal; esto promueve aun más erosión. En síntesis, la reducción de estructura que producen el pastoreo y el fuego en los bosques redunda en una pérdida de la capacidad del suelo de almacenar agua.

En términos generales, nuestros resultados son coincidentes con trabajos previos en la zona de estudio, donde sitios con vegetación menos estructurada presentaron menor profundidad y materia orgánica y mayor compactación del suelo (Renison et al. 2010; Vaieretti et al. 2010; Cingolani et al. 2013, 2014). Con este trabajo aportamos evidencia adicional al haber restringido el efecto de la topografía y abarcado la variabilidad completa en la estructura de la vegetación y, a su vez, sumamos dos variables clave relacionadas con la capacidad de almacenamiento del agua de los bosques de $P$. australis de las sierras de Córdoba, producto de la degradación por el disturbio histórico o de los procesos de recuperación posteriores. Asimismo, nuestros resultados confirman lo hipotetizado en trabajos previos en los que se sugería que los céspedes y pedregales de estos ecosistemas podrían tener una tasa de infiltración reducida por alteraciones en las propiedades físicoquímicas del suelo (Cingolani et al. 2003; Vaieretti et al. 2010), en comparación a los suelos de los bosques y pajonales que tendrían mayores oportunidades de infiltración (Cingolani et al. 2015).

En resumen, a medida que el sistema vegetación-suelo de los bosques de $P$. australis del centro de Argentina es menos alterado por el uso histórico y actual, presenta mayores tasas de infiltración y profundidad del suelo; esto sugiere una mayor capacidad para almacenar agua. Nuestros resultados van en línea con lo reportado por otros trabajos que destacan el efecto negativo del disturbio por el pastoreo y el fuego sobre estas propiedades (Noy-Meir 1973; Abdel-Magid et al. 1987; Boone Kauffman et al. 2004; Yong-Zhong et al. 2005). A su vez, remarcando la pronunciada estacionalidad de las precipitaciones en este sistema, sugerimos que a menor disturbio, mayor potencialidad de estos sitios para regular la humedad del suelo y almacenar, en mayor medida, agua freática que los sitios con características opuestas (Bruijnzeel 1988, 2004; Roa-García et al. 2011), lo que los convierte en potenciales reservorios de agua en el paisaje (Cingolani et al. 2015). Resaltamos la necesidad de futuros trabajos en este ecosistema que evalúen la dinámica hídrica anual a escala de sitio comprendiendo la variabilidad completa existente en el sistema vegetación-suelo y que 
pongan a prueba la potencialidad de nuestros resultados.

Agradecimientos. Agradecemos a Carolina Furey, Lucrecia Lipoma, Laura Fraschina, Eugenia Marcotti y Cecilia Ferrero por su asistencia en mediciones de campo. Agradecemos también a la Administración de Parques Nacionales que nos permitió desarrollar este trabajo en el Parque Nacional
Quebrada del Condorito y a la Familia Palmieri que nos permitió realizar mediciones en su campo privado. Por último, agradecemos a dos revisores anónimos y al editor asociado, cuyos comentarios mejoraron la calidad del manuscrito. Este trabajo fue realizado gracias al financiamiento de CONICET (PIP 112-200801-01458 y PIP 112-201201-00164) y FONCyT (PICT-2012-1357).

\section{REFERENCIAS}

Abdel-Magid, A. H., G. E. Schuman, and R. H. Hart. 1987. Soil bulk density and water infiltration as affected by grazing systems. J Range Manage 40:307-309.

Altesor, A., G. Piñeiro, F. Lezama, R. B. Jackson, M. Sarasola and J. M. Paruelo 2006. Ecosystem changes associated with grazing in subhumid South American grasslands. J Veg Sci 17(3):323-332.

APN (Administración de Parques Nacionales). 2007. Plan de Manejo del Parque Nacional Quebrada del Condorito. Reserva Hídrica Provincial de Achala. Editorial APN, Ciudad Autónoma de Buenos Aires, Argentina.

Aronson J., D. Renison, J. O. Rangel-Ch., S. Levy-Tacher, C. Ovalle, and A. Del Pozo. 2007. Restauración del Capital Natural: sin reservas no hay bienes ni servicios. Revista Ecosistemas, 16(3):1-10.

Belsky, A. J., and D. M. Blumenthal. 1997. Effects of Livestock Grazing on Stand Dynamics and Soils in Upland Forests of the Interior West. Conserv Biol 11(2):315-327.

Bernardi, R. E., M. Holmgren, M. Arim, and M. Scheffer. 2016. Why are forests so scarce in subtropical South America? The shaping roles of climate, fire and livestock. Forest Ecol Manag 363:212-217.

Beven, K., and P. Germann. 2013. Macropores and water flow in soils revisited. Water Resource Res 49(6):3071-3092.

Bonell, M., B. K. Purandara, B. Venkatesh, J. Krishnaswamy, H. A. K. Acharya, U. V. Singh, R. Jayakumar, and N. Chappell. 2010. The impact of forest use and reforestation on soil hydraulic conductivity in the Western Ghats of India: implications for surface and sub-surface hydrology. J Hydrol 391(1):47-62.

Boone Kauffman, J., A. S. Thorpe, and E. J. Brookshire. 2004. Livestock Exclusion and Belowground Ecosystem Responses in Riparian Meadows of Eastern Oregon. Ecol Appl 14(6):1671-1679.

Bouwer, H. 1986. Intake rate: cylinder infiltrometer. En: Klute, A. (Ed.). Methods of Soil Analysis: Part 1-Physical and Mineralogical Methods. 2nd Ed. Madison: American Society of Agronomy and Soil Science Society of America. Pp. 825-844.

Bremner, J. M. 1996. Nitrogen - Total. Pp. 961-1010 en D. L. Sparks, A. L. Page, P. A. Helmke, R. H. Loeppert, P. N. Soltanpour, M. A. Tabatabai, C. T. Johnston and M. E. Sumner (eds.). Methods of Soil Analysis. Part 3 - Chemical Methods. Madison, Wisconsin, USA.

Bruijnzeel, L. A. 1988. (De)Forestation and dry season flow in the tropics: a closer look. J Trop Forest Sci 1(3):229243.

Bruijnzeel, L. A. 2004. Hydrological functions of tropical forests: not seeing the soil for the trees? Agr Ecosyst Environ 104(1):185-228.

Cabido, M., R. Breimer, and G. Vega. 1987. Plant communities and associated soil types in a high plateau of the Córdoba mountains, central Argentina. Mt Res Dev 7:25-42.

Canadell, J., R. B. Jackson, J. B. Ehleringer, H. A. Mooney, O. E. Sala, and E. D. Schulze. 1996. Maximum rooting depth of vegetation types at the global scale. Oecologia 108(4):583-595.

Cingolani, A. M., M. R. Cabido, D. Renison, and V. Solís Neffa. 2003. Combined effects of environment and grazing on vegetation structure in Argentine granite grasslands. J Veg Sci 14(2):223-232.

Cingolani, A. M., D. Renison, M. R. Zak, and M. R. Cabido. 2004. Mapping vegetation in a heterogeneous mountain rangeland using Landsat data: an alternative method to define and classify land-cover units. Remote Sens Environ 92(1):84-97.

Cingolani, A. M., D. Renison, P. A. Tecco, D. E. Gurvich, and M. Cabido. 2008. Predicting cover types in a mountain range with long evolutionary grazing history: a GIS approach. J Biogeogr 35(3):538-551.

Cingolani, A. M., M. V. Vaieretti, M. A. Giorgis, N. La Torre, J. I. Whitworth-Hulse, and D. Renison. 2013. Can livestock and fires convert the sub-tropical mountain rangelands of central Argentina into a rocky desert? Rangeland J 35(3): 285-297.

Cingolani, A. M., M. V. Vaieretti, M. A. Giorgis, M. Poca, P. A. Tecco, and D. E. Gurvich. 2014. Can livestock grazing maintain landscape diversity and stability in an ecosystem that evolved with wild herbivores? Perspect Plant Ecol Evol Syst 16(4):143-153.

Cingolani, A. M., M. Poca, M. A. Giorgis, M. V. Vaieretti, D. E. Gurvich, J. I. Whitworth-Hulse, and D. Renison. 2015. Water provisioning services in a seasonally dry subtropical mountain: Identifying priority landscapes for conservation. J Hydrol 525:178-187.

Colladon, L. 2004. Temperaturas medias mensuales. Cuenca del río San Antonio, Sistema del Río Suquía, Provincia de Córdoba. Instituto Nacional del Agua y del Ambiente (INA) y Centro de Investigaciones de la Región Semiárida 
(CIRSA). Córdoba, Argentina.

Colladon, L. 2014. Anuario pluviométrico 1992-2012. Cuenca del Río San Antonio, Sistema del Río Suquía, Provincia de Córdoba. Córdoba, Argentina. Instituto Nacional del Agua y del Ambiente (INA) y Centro de Investigaciones de la Región Semiárida (CIRSA). Córdoba, Argentina.

Conti, G., L. Enrico, F. Casanoves, and S. Díaz. 2013. Shrub biomass estimation in the semiarid Chaco forest: a contribution to the quantification of an underrated carbon stock. Ann For Sci 70(5):515-524.

Di Rienzo, J. A., F. Casanoves, M. G. Balzarini, L. González, and M. Tablada. 2013. InfoStat versión 2013. Grupo InfoStat, FCA, Universidad Nacional de Córdoba, Argentina. URL: www.infostat.com.ar.

Germer, S., C. Neill, A. V. Krusche, and H. Elsenbeer. 2010. Influence of land-use change on near-surface hydrological processes: undisturbed forest to pasture. J Hydrol 380(3):473-480.

Giorgis, M. A., A. M. Cingolani, I. Teich, D. Renison, and I. Hensen. 2010. Do Polylepis australis trees tolerate herbivory? Seasonal patterns of shoot growth and its consumption by livestock. Plant Ecol 207(2):307-319.

Gómez-Plaza, A., M. Martýnez-Mena, J. Albaladejo, and V. M. Castillo. 2001. Factors regulating spatial distribution of soil water content in small semiarid catchments. J Hydrol 253(1):211-226.

Kirkham, M. B. 2005. Principles of soil and plant water relations. Academic Press.

McCune, B., and M. J. Mefford. 1999. PC-ORD: multivariate analysis of ecological data. Version 4.01. MjM software design. Gleneden Beach, Oregon, USA

Milchunas, D. G., and W. K. Lauenroth. 1993. Quantitative effects of grazing on vegetation and soils over a global range of environments. Ecol Monogr 63(4):327-366.

Neary, D. G., G. G. Ice, and C. R. Jackson. 2009. Linkages between forest soils and water quality and quantity. Forest Ecol Manag 258(10):2269-2281.

Nelson, D. W., and L. E. Sommers. 1996. Total Carbon, Organic carbon, and Organic Matter. Pp. 961-1010 en D. L. Sparks, A. L. Page, P. A. Helmke, R. H. Loeppert, P. N. Soltanpour, M. A. Tabatabai, C. T. Johnston and M. E. Sumner (eds.). Methods of Soil Analysis. Part 3 - Chemical Methods. Madison, Wisconsin, USA.

Noy-Meir, I. 1973. Desert ecosystems: environment and producers. Annu Rev Ecol Syst 4:25-51.

Pimentel, D., C. Harvey, P. Resosudarmo, and K. Sinclair. 1995. Environmental and economic costs of soil erosion and conservation benefits. Science 267(5201):1117.

Poca, M., M. V. Vaieretti, A. M. Cingolani, and N. Pérez-Harguindeguy. 2015. Scaling-up from species to ecosystems: How close can we get to actual decomposition? Acta Oecol 64:1-9.

Renison, D., I. Hensen, R. Suarez, and A. M. Cingolani. 2006. Cover and growth habit of Polylepis woodlands and shrublands in the mountains of central Argentina: human or environmental influence? J Biogeogr 33(5):876-887.

Renison, D., I. Hensen, R. Suarez, A. M. Cingolani, P. Marcora, and M. A. Giorgis. 2010. Soil conservation in Polylepis mountain forests of Central Argentina: Is livestock reducing our natural capital? Austral Ecol 35(4):435-443.

Renison, D., M. P. Chartier, M. Menghi, P. Marcora, R. C. Torres, M. Giorgis, I. Hensen, and A. M. Cingolani. 2015. Spatial variation in tree demography associated to domestic herbivores and topography: Insights from a seeding and planting experiment. Forest Ecol Manag 335:139-146.

Roa-García, M. C., S. Brown, H. Schreier, and L. M. Lavkulich. 2011. The role of land use and soils in regulating water flow in small headwater catchments of the Andes. Water Resour Res 47(5). DOI: 10.1029/2010WR009582

Teich, I., A. M. Cingolani, D. Renison, I. Hensen, and M. A. Giorgis. 2005. Do domestic herbivores retard Polylepis australis Bitt. woodland recovery in the mountains of Córdoba, Argentina? Forest Ecol Manag 219(2):229-241.

Throop, H. L., S. R. Archer, H. C. Monger, and S. Waltman. 2012. When bulk density methods matter: Implications for estimating soil organic carbon pools in rocky soils. J Arid Environ 77:66-71.

Torres, R. C., D. Renison, I. Hensen, R. Suarez, and L. Enrico. 2008. Polylepis australis' regeneration niche in relation to seed dispersal, site characteristics and livestock density. Forest Ecol Manag 254(2):255-260.

USDA (United States Department of Agriculture). 1999. Soil Quality Test Kit Guide. Agricultural Research Service, Natural Resources Conservation Service, Soil Quality Institute.

Vaieretti, M. V., A. M. Cingolani, N. Pérez-Harguindeguy, D. E. Gurvich, and M. Cabido. 2010. Does decomposition of standard materials differ among grassland patches maintained by livestock? Austral Ecol 35(8):935-943.

von Müller A. R., D. Renison, and A. M. Cingolani. 2017. Cattle landscape selectivity is influenced by ecological and management factors in a heterogeneous mountain rangeland. Rangeland J RJ15114.

Wondzell, S. M., and J. G. King. 2003. Post-fire erosional processes in the Pacific North-west and Rocky Mountain regions. Forest Ecol Manag 178:75-87. DOI:10.1016/S0378-1127(03)00054-9

Yong-Zhong, S., L. Yu-Lin, C. Jian-Yuan, and Z. Wen-Zhi. 2005. Influences of continuous grazing and livestock exclusion on soil properties in a degraded sandy grassland, Inner Mongolia, northern China. Catena 59(3):267-278.

Zhang, B., Y. S. Yang, and H. Zepp. 2004. Effect of vegetation restoration on soil and water erosion and nutrient losses of a severely eroded clayey Plinthudult in southeastern China. Catena 57(1):77-90. 